\title{
Iron-deficiency anemia due to massive trichobezoar
}

\author{
Jo-Anne Janson ${ }^{1}$, Colleen A.L. Vaessens ${ }^{1}$, Jan H.M.B Stoot ${ }^{2}$, Eric T.P. Keulen ${ }^{3}$, Philippe P.R. Rosias*1 \\ ${ }^{1}$ Department of Pediatrics, Zuyderland Medical Centre, Sittard, the Netherlands \\ ${ }^{2}$ Department of Surgery, Zuyderland Medical Centre, Sittard, the Netherlands \\ ${ }^{3}$ Department of Gastroenterology, Zuyderland Medical Centre, Sittard, the Netherlands
}

Received: January 28, 2016

Accepted: February 19, 2016

Online Published: March 14, 2016

DOI: $10.5430 /$ crim.v3n2p15

URL: http://dx.doi.org/10.5430/crim.v3n2p15

\begin{abstract}
This case-report describes a 15-year-old girl who presented at the outpatient department with iron-defiency anemia. Physical examination showed a mass in the left upper quadrant. Additional ultrasound, MRI and gastroscopy revealed a large trichobezoar and extensive bleeding ulcera in the stomach. The trichobezoar was successfully removed during surgery. Patient recovery was complete, and psychiatric treatment was initiated to prevent relapse of trichotillomania and trichophagia.
\end{abstract}

Key Words: Iron-deficiency anemia, Bleeding ulcera, Trichobezoar, Trichophagia, Surgery

\section{INTRODUCTION}

The most common cause of microcytic anemia in children is iron-deficiency anemia due to poor dietary intake of iron. ${ }^{[1]}$ However, other causes should also be considered, such as malabsorption, hereditary disorders and gastro-intestinal losses. This case report presents an uncommon cause of iron-deficiency anemia.

\section{Case presentation}

A 15-year-old Caucasian girl came to the outpatient pediatric department because of persistent iron-deficiency anemia of unknown origin. Medical history was not remarkable, nor suggestive for any cause of anemia. The patient did not report weight loss, gastro-intestinal blood loss, heavy menstruation or lack of iron from dietary intake. Furthermore, there was no family history of clotting disorders or hereditary anemia. She did complain of progressive fatigue and pain in the left upper abdominal quadrant, which existed for a few months. Physical evaluation showed a BMI of $16.4 \mathrm{~kg} / \mathrm{m}^{2}$ $(-0.84 S D)$ with normal vital signs, pale skin and mucosa, no jaundice and tenderness of the left upper abdominal quadrant without signs of peritoneal irritation. Local deep palpation was suggestive of a mass.

Additional laboratory tests showed a hemoglobin of 5.0 $\mathrm{mmol} / \mathrm{L}$, hematocrit $0.28 \mathrm{~L} / \mathrm{L}, \mathrm{MCV} 64 \mathrm{fl}$, RDW $17.6 \%$, leukocyte count $6.0 \times 10^{9} / \mathrm{L}$, leukocyte differentiation: 3.41 $\times 10^{9} / \mathrm{L}$ neutrophil granulocytes, $1.51 \times 10^{9} / \mathrm{L}$ lymphocytes, $0.58 \times 10^{9} / \mathrm{L}$ monocytes, $0.01 \times 10^{9} / \mathrm{L}$ basophils, $0.44 \times$ $10^{9} / \mathrm{L}$ eosinophils, platelets $356 \times 10^{9} / \mathrm{L}$, iron $6 \mu \mathrm{mol} / \mathrm{L}$, ferritin $12 \mu \mathrm{g} / \mathrm{L}$, erythrocyte sedimation rate $13 \mathrm{~mm} / \mathrm{h}, \mathrm{C}$ reactive protein $<1 \mathrm{mg} / \mathrm{L}$, and screening for celiac disease was negative.

Bone marrow cell morphology and iron staining were not yet scheduled in expectation of additional imaging of the mass felt in the left upper abdominal quadrant. Abdominal ultrasound revealed a large round hyperreflective structure in the stomach and a thickened gastric wall of $10 \mathrm{~mm}$ (see Figure 1). Additional MRI of the abdomen showed a large loose bean-shaped intra-gastric structure (diameter $15.5 \mathrm{~cm}$ ),

\footnotetext{
* Correspondence: Phippe P.R. Rosias; Email: p.rosias@ orbisconcern.nl; Address: Zuyderland Medical Centre, Sittard-Geleen, 6130 MB Sittard, the Netherlands.
}

Published by Sciedu Press 
covering more than half of the gastric content, without signs of metastasis or enlarged lymph nodes (see Figure 2).

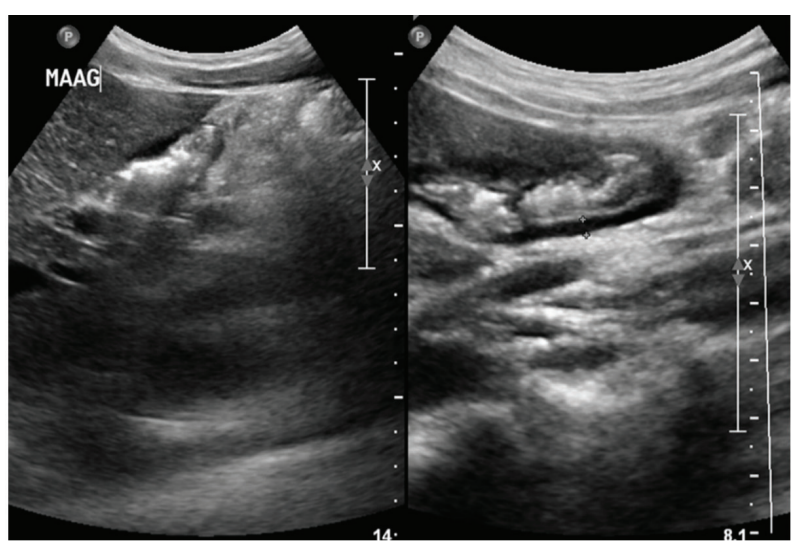

Figure 1. Ultrasound of stomach (Note the hyperreflective structure in the stomach)
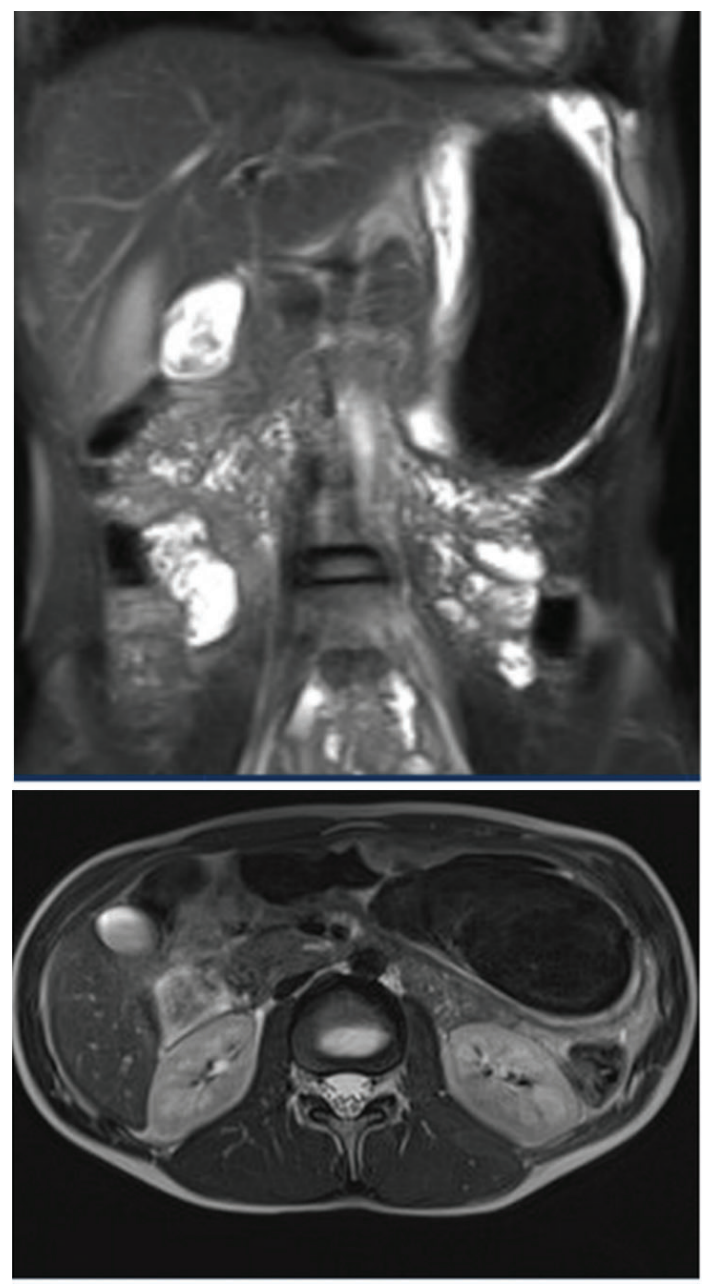

Figure 2. MRI-abdomen (Note the large loose bean-shaped intra-gastric structure [diameter $15.5 \mathrm{~cm}$ ], covering more than half of the gastric content)

Because of these findings, a gastroscopy was performed and revealed a large trichobezoar with extensive bleeding ulcera along the large curvature (see Figure 3). Extensive bleeding from these ulcera was the most likely cause of iron-deficiency anemia. Surgery was planned within a week. At first, endoscopical removal was tried, but failed due to the large size. Hence, endoscopic surgery was converted to laparotomy. Eventually a bezoar with a weight of more than 0.5 kilogram was removed successfully (see Figure 4).
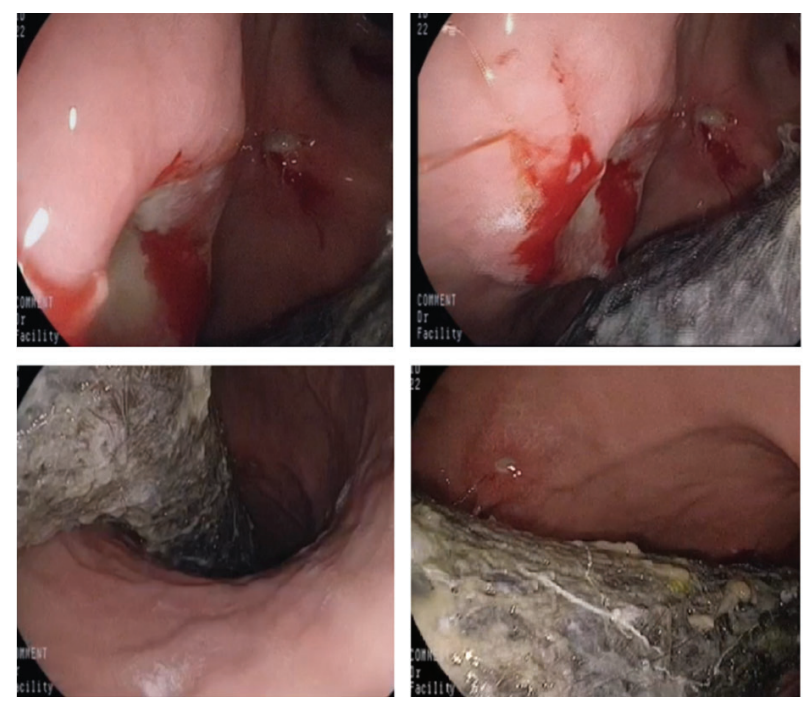

Figure 3. Gastroscopy (Large bleeding ulcera in great curvature of stomach, large solid trichobezoar)

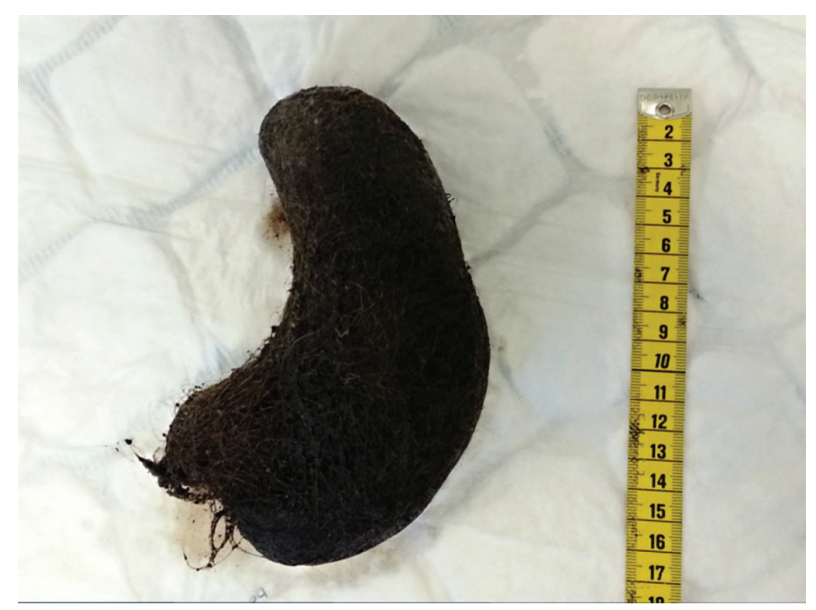

Figure 4. Trichobezoar after surgical removal

Due to this finding it became clear to the parents that their daughter still suffered from trichotillomania (pulling out hairs) and trichophagia (eating hair). During childhood she had a history of trichotillomania and trichophagia, but her parents thought it stopped many years ago. Meanwhile, she did not demonstrate abnormal behavior, and there were no physical signs such as alopecia. Postoperatively, psychiatric 
treatment was reinitiated to prevent relapse and she was regularly seen on an outpatient basis by a pediatric psychiatrist and psychologist. Initial treatment also consisted of oral iron suppletion and an oral proton pump inhibitor. Shorttime follow up showed absence of symptoms and rapid normalization of hemoglobin. At a check-up 17 months after surgery, she was doing well and did not have any symptoms of fatigue or (stomach) pain. Laboratory values remained normal: Hemoglobin $8.8 \mathrm{mmol} / \mathrm{L}$, hematocrit $0.40 \mathrm{mmol} / \mathrm{L}$, MCV 93 fl, RDW $12.2 \%$, leukocytes $6.3 \times 10^{9} / \mathrm{L}$, thrombocytes 281 $\times 10^{9} / \mathrm{L}$. According to the patient, the mental impact of this specific diagnosis was positively in such a way that trichotillomania and trichophagia stopped and further psychiatric treatment was not required.

\section{Discussion}

Gastric bezoar is defined as a foreign body resulting from accumulation of ingested material, commonly found as a hard mass in the stomach. ${ }^{[2]}$ There are several forms, such as phytobezoar (vegetable), thichobezoar (hair) or pharmacobe- zoar (medication). ${ }^{[2]}$ In some rare cases, there is extension of the trichobezoar into the small intestine, a condition labeled as Rapunzel syndrome, referring to the Grimm brothers' tale about the long-haired Rapunzel. ${ }^{[3]}$ Trichobezoars are almost exclusively seen in young females with psychiatric comorbidity. ${ }^{[4]}$ Most patients are asymptomatic for many years. Most common symptoms are abdominal pain, nausea, vomiting, anorexia and weight loss. ${ }^{[2]}$ Iron-deficiency anemia is also common in patients with trichobezoars, and is the direct outcome of bleeding gastric ulcera due to pressure by massive trichobezoar. ${ }^{[5]}$ Early diagnosis and treatment of trichobezoars is of great importance and may avoid later fatal complications. It is recommended that general practitioners, pediatricians and doctors working in the field of internal medicine are aware of this uncommon condition, which can also present as iron-deficiency anemia without suggestive symptoms.

\section{CONFLICTS OF INTEREST DISClOSURE}

The authors have declared no conflicts of interest.

\section{REFERENCES}

[1] Irwin JJ, Kirchner JT. Anemia in children. American Family Physician. 2001; 64(8): 1379-1386. PMid:11681780

[2] Debakey M, Oschner A. Bezoars and concretions, comprehensive review of literature with analysis of 303 collected cases and presentation of 8 additional cases. Surgery. 1939; 5: 132-160.

[3] Vaughn ED Jr, Sawyers JL, Scott HW Jr. The Rapunzel syndrome: an unusual complication of intestinal bezoar. Surgery. 1968; 63: 339-43.
[4] Gorter RR, Kneepkens CMF, Mattens ECJL, et al. Management of trichobezoar: case report and literature review. Pediatr Surg Int. 2010; 26: 457-463. PMid:20213124 http://dx.doi.org/10.10 07/s00383-010-2570-0

[5] Demirel AH, Akbal E, Koklu S, et al. An unusual cause of iron deficiency anemia and abdominal pain in a young female. Am Surg. 2011; 77: 127-8. PMid:21396329 